\title{
Preventing hospital admissions for COPD: role of physical activity
}

\section{D L Morgan}

\section{There is increasing evidence that non-pharmacological interventions including physical activity may prevent hospital admissions for COPD.}

A hospital admission or readmission for an exacerbation of chronic obstructive pulmonary disease (COPD) is bad news for everyone. For the patient it may signal the beginning of the terminal phase of the illness. For the health services it is a significant component of the cost of care for a condition that is increasingly burdensome. In recent years knowledge has been growing about the important role of the exacerbation in the deteriorating progress of COPD. The last few years of life with COPD may be characterised by repeated episodes of illness culminating in hospital admission. Exacerbations only lead to hospital admission in about $16 \%$ of cases, but their increasing frequency is associated with declining state of health. ${ }^{1}$

In UK hospitals emergency inpatient admission is the largest component of the total cost of respiratory disease to the NHS that amounts to over $£ 2.5$ billion. ${ }^{2}$ In recent years attention has focused on reducing the cost and impact of hospital admissions for COPD. The emphasis of the strategy to date has been on admission avoidance and early supported discharge schemes. ${ }^{3-5}$ These have had some success in curtailing admissions and reducing lengths of hospital stay, and are beginning to be introduced more widely into clinical practice. Contrary to popular perception, COPD is not a stable condition and there are inevitably day to day variations in both symptoms and ability to function. The periodic episodes of worsening are known as exacerbations, although a precise agreed definition is lacking. However, the meaning of the term is generally understood and a working appreciation of the impact of these events is becoming clear. Not all episodes of exacerbation lead to hospital admission, but if these can be contained or prevented by the patient or primary care services, then the burden of COPD will be reduced. A study of the factors that can prevent hospital admission in these circumstances would therefore make an important and valuable contribution.

This issue of Thorax contains the third major publication from a group in Barcelona (EFRAM) that has been examining the factors associated with hospital admission. ${ }^{6}$ This latest paper is the report of a prospective study that examines the risk factors for readmission to hospital for exacerbations of COPD. In previous publications the group have described the prevalence and relative risk of modifiable risk factors for hospital admission. ${ }^{78}$ The initial study population was a sample of 404 patients who were admitted to hospital in Barcelona over a period of 1 year. ${ }^{7}$ During the admission the patients completed a questionnaire and 353 later underwent spirometric tests and arterial blood gas measurements. Identification of risk factors that were potentially amenable to modification showed that lack of rehabilitation and poor inhaler technique were the most frequent associations. Other important factors were continued smoking, inadequate oxygen prescription, and lack of influenza immunisation. In the second paper the authors reported a casecontrol study of the factors associated with hospital admission in a subsample of the original cohort. ${ }^{8}$ The control subjects were patients with COPD who had had a previous admission but were stable at the time of the comparison. The only apparent risk factors found in 86 pairs of cases and controls were a previous history of three or more admissions, lower $\mathrm{FEV}_{1}$, and underprescription of oxygen. Perversely, continued smoking appeared to convey an advantage and inhaled corticosteroids offered no benefit. Some other potentially modifiable factors including rehabilitation could not be tested because of the very small numbers receiving it. In their conclusion the authors acknowledged that the casecontrol methodology had limitations in terms of selection bias and small numbers and that a prospective cohort study was required.

The latest paper describes a prospective study of the risk of readmission in a cohort of 340 patients from the original study population who were followed for over 1 year after their index admission. ${ }^{6}$ At the end of the study $63 \%$ of the patients had been readmitted and 29\% had died, suggesting that previous admission is an important risk factor.
Other expected risk factors were lower FEV and hypoxaemia. One unexpected finding was that a high level of usual physical activity was associated with a $46 \%$ reduction in the risk of admission. The activity profile was obtained through self-reporting and no objective test of exercise capacity was made. Nevertheless, the association was very strong. Once again the authors could not test the influence of rehabilitation because the numbers were too small. Other factors weakly associated with increased admission were supervision by a respiratory specialist, oral corticosteroids, and anticholinergic drugs. These latter associations are unlikely to be causal and probably reflect confounding by severity of disease.

Similar findings were reported by the British Thoracic Society and Royal College of Physicians' audit of admission for acute exacerbation of COPD. ${ }^{9}$ In this audit of 1400 admissions two thirds of the patients had had a previous admission for COPD and one third had had a similar episode in the previous 4 months. In a follow up audit of readmission within 3 months, poor performance status was a predictor of mortality at the first admission but not readmission. ${ }^{10}$ Once again the best predictors of admission were low $\mathrm{FEV}_{1}$ and previous admission.

The pattern of hospital readmission may be affected by both patient and healthcare delivery factors. It could be that general practitioners find it easier to admit the patient to hospital than to deal with the exacerbation at home. However, high rates of readmission to hospital are common to both studies from different healthcare systems. The implication of the latest EFRAM study is that the risk of readmission to hospital for COPD can be reduced by improving spontaneous domestic activity and thereby breaking the cycle of hospital dependency. This may be true but, so far, trials of pulmonary rehabilitation that improve exercise capacity have been unable to show a reduction in hospital admission. However, they have shown a reduction in the length of stay once admitted. ${ }^{11}$ It is possible that rehabilitation may reduce hospital admissions but studies with an appropriately sensitive design have not been performed. In addition, the capacity to provide rehabilitation in most countries is so poor that it has never been testable. In the British Thoracic Society audit only $3 \%$ of the patients were recommended for rehabilitation, while in Spain only $14 \%$ of the study group had received it. An alternative explanation for the results of the study is that patients with less severe COPD simply feel better, do more, cope better, and are not admitted so frequently. It is known that physical performance, as reflected by a walking test and functional performance questionnaire, is a strong 
predictor of outcome in COPD. ${ }^{12}$ The answer to the important question of whether the prognosis can be altered by increasing functional performance is not known. The arguments are somewhat analogous to those about the presence of nutritional depletion in severe COPD, where a low body mass index is associated with increased mortality but attempts to change the situation by supplementation have been ineffective..$^{13} 14$ In the case of physical function, however, it is clear that the simple intervention of exercise training will improve performance and have a prolonged effect on lifestyle. ${ }^{15}$ It would therefore be reasonable to test the hypothesis that rehabilitation can reduce hospital readmission in an appropriately susceptible group.

In the UK a hospital admission for COPD generally involves a length of stay of 8 days. ${ }^{9}$ From the patient's perspective, there is a flurry of attention at the beginning of the admission followed by 7 days of observed inactivity. As result, patients may leave hospital less well equipped for independent life than when they were admitted. Characteristically, our hospital and community services are attuned to dealing with one crisis but make little attempt to prevent the next. The huge financial costs of hospital admissions for exacerbations of COPD deserve some exploration of the value of actions that may prevent the initial admission or reduce the frequency of readmission. The cost of drug treatment for these patients is second only to the cost of hospitalisation, but to adhere to the 20th century view that drug treatment alone will provide all the answers is a delusion. The diverse factors associated with advanced COPD require a multimodality approach by a multiprofessional team. There is now increasing evidence that non-pharmacological interventions may play a major role. Let us hope that this evidence is now persuasive enough to promote appropriate investment in research and services for this neglected condition.

Thorax 2003;58:95-96

\section{Author's affiliation}

M D L Morgan, Institute for Lung Health, Department of Respiratory Medicine and Thoracic Surgery, University Hospitals of Leicester, Glenfield Hospital, Leicester LE3 9QP, UK; mike.morgan@uhl-tr.nhs.uk

\section{REFERENCES}

1 Seemungal TA, Donaldson GC, Paul EA, et al. Effect of exacerbation on quality of life in patients with chronic obstructive pulmonary disease. Am J Respir Crit Care Med 1998;157:1418-22.

2 British Thoracic Society. The burden of lung disease. London: British Thoracic Society, 2002.

3 Gravil JH, Al Rawas OA, Cotton MM, et al. Home treatment of exacerbations of chronic obstructive pulmonary disease by an acute respiratory assessment service. Lancet 1998;351:1853-5.

4 Davies L, Wilkinson M, Bonner S, et al. "Hospital at home" versus hospital care in patients with exacerbations of chronic obstructive pulmonary disease: prospective randomised controlled trial. BM 2000;321:1265-8

5 Skwarska E, Cohen G, Skwarski KM, et al. Randomized controlled trial of supported discharge in patients with exacerbations of chronic obstructive pulmonary disease. Thorax 2000;55:907-12.

6 Garcia-Aymerich J, Farrero E, Félez MA, et al. Risk factors of readmission to hospital for a COPD exacerbation: a prospective study. Thorax 2003;58:100-5.

7 Garcia-Aymerich J, Barreiro E, Farrero E, et al. Patients hospitalized for COPD have a high prevalence of modifiable risk factors for exacerbation (EFRAM study). Eur Respir J 2000;16:1037-42

8 Garcia-Aymerich J, Monso E, Marrades RM, et al. Risk factors for hospitalization for a chronic obstructive pulmonary disease exacerbation. EFRAM study. Am J Respir Crit Care Med 2001;164:1002-7.

9 Roberts CM, Ryland I, Lowe D, et al. Audit of acute admissions of COPD: standards of care and management in the hospital setting. Eur Respir J 2001;17:343-9.

10 Roberts CM, Lowe $D$, Bucknall CE, et al. Clinical audit indicators of outcome following admission to hospital with acute exacerbation of chronic obstructive pulmonary disease. Thorax 2002;57:137-41.

11 Griffiths TL, Burr ML, Campbell IA, et al. Results at 1 year of outpatient multidisciplinary pulmonary rehabilitation: a randomised controlled trial. Lancet 2000;355:362-8.

12 Bowen JB, Votto JJ, Thrall RS, et al. Functional status and survival following pulmonary rehabilitation. Chest 2000;1 18:697-703.

13 Landbo C, Prescott E, Lange P, et al. Prognostic value of nutritional status in chronic obstructive pulmonary disease. Am J Respir Crit Care Med 1999;160:1856-61.

14 Ferreira IM, Brooks D, Lacasse $Y$, et al. Nutritional support for individuals with COPD: a meta-analysis. Chest 2000; 1 17:672-8.

15 British Thoracic Society. Statement on pulmonary rehabilitation. Thorax 2001;56:827-34.

\section{The new front line treatment for malignant pleural mesothelioma?}

\section{J P C Steele}

The presentation at the recent meeting of the American Society of Clinical Oncology of the results of the largest phase III trial in malignant pleural mesothelioma has aroused renewed interest in the treatment of this cancer. What are the implications for the ongoing UK mesothelioma trial?

M alignant pleural mesothelioma has become a mainstream cancer. This is partly due to the increasing incidence, ${ }^{12}$ but is also a result of the advances being made in its treatment. This summer the American Society of Clinical Oncology Annual Meeting plenary session included a clinical research paper on malignant pleural mesothelioma for the first time. ${ }^{3}$ In fact, this may well have been the first oral presentation on the disease at this important international meeting. The reason for the increased interest is that the study presented is the largest phase III randomised trial reported in malignant pleural mesothelioma. The trial, which recruited internationally and was led by researchers at the University of Chicago, showed a positive clinical benefit for an experimental arm based on a novel chemotherapy drug. But what do these data mean for respiratory physicians, oncologists and patients, and is this a definitive result? What effect, if any, does this trial result have on the ongoing UK mesothelioma trial?

The University of Chicago multicentre trial compared a combination of pemetrexed and cisplatin chemotherapy with a control arm of single agent cisplatin. Pemetrexed is a new cytotoxic drug that inhibits several folate dependent reactions that are essential for cell proliferation, hence its previous name "multitargeted antifolate". ${ }^{4}$ Its primary target is thymidylate synthase, but it also inhibits folate dependent enzymes involved in purine synthesis. It is related to the existing cytotoxic drugs methotrexate, 5-fluorouracil, and raltitrexed. Phase I and II data had suggested a dose for a 21 day cycle of $500-600 \mathrm{mg} / \mathrm{m}^{2}$ pemetrexed and $75-100 \mathrm{mg} / \mathrm{m}^{2}$ cisplatin, with both drugs being administered on the same day. ${ }^{56}$ The investigators of the phase III trial chose doses of $75 \mathrm{mg} / \mathrm{m}^{2}$ cisplatin and $500 \mathrm{mg} / \mathrm{m}^{2}$ pemetrexed for the experimental arm. Patients randomised to the control arm received $75 \mathrm{mg} / \mathrm{m}^{2}$ cisplatin. All patients were treated every 3 weeks. 
A total of 472 patients with malignant pleural mesothelioma were recruited between 1998 and 2002. All had good performance status (Karnofsky score 70-100\%). After an initial accrual period, four drug related deaths from febrile neutropenia were noted (three in the experimental arm and one in the control arm). These deaths were linked to raised homocysteine levels and it was decided to give all patients folic acid supplements and vitamin B12 to counter this. This measure appeared to reduce the toxicity of the chemotherapy in both arms of the study. The researchers reported that, for the complete cohort of patients, the combination therapy significantly lengthened the time to disease progression ( 5.7 months $v 3.9$ months; $\mathrm{p}=0.001$ ) and overall survival (12.1 months $v 9.3$ months; $p=0.020)$. For patients receiving full vitamin supplementation, the overall survival for those treated with pemetrexed and cisplatin was 13.3 months compared with 10.0 months for the control patients $(p=0.051)$. The study organisers concluded that the combination of pemetrexed and cisplatin with folic acid and vitamin B12 should now be considered the "standard front line therapy for patients with malignant pleural mesothelioma".

In the UK the British Thoracic Society has recently completed the pilot phase of a randomised trial of chemotherapy for patients with malignant pleural mesothelioma. ${ }^{7}$ The trial-known as "MESO-1"started as a feasibility study because the investigators wanted to determine which of two quality of life instruments was more appropriate. MESO-1 contained a multiple randomisation option such that patients and their oncologists could choose to be randomised between active symptom control (ASC) versus one of two chemotherapy regimens or between the chemotherapy regimens only. The chemotherapy regimens chosen were single agent vinorelbine for 12 weeks and MVP (mitomycin C, vinblastine and cisplatin) for four 21 day cycles. These regimens were chosen because they both give a response rate of approximately $20 \%$ and have proven quality of life benefit in a substantial proportion of patients. ${ }^{89}$
Following completion of the pilot study, the trial has been granted full support from Cancer Research UK and the National Cancer Research Network and is now designated "MSOl". This three arm phase III trial aims to randomise 840 patients with malignant pleural mesothelioma into one of three arms: ASC without chemotherapy; ASC with vinorelbine chemotherapy; and ASC with MVP chemotherapy. The main end points of MSOl are overall survival, symptom palliation, quality of life, toxicity, response, and recurrence. One hundred and fifty patients from the pilot study who were randomised between all three arms will be included in the MSOl analysis.

The important question is whether the MSOl trial is still ethical in the light of the new data on pemetrexed with cisplatin. I think the answer is "yes". The University of Chicago trial, although promising and an important step in the advancement of knowledge of mesothelioma, can be criticised. Firstly, what was the rationale for the control arm? Few physicians would recommend single agent cisplatin in a dose of $75 \mathrm{mg} / \mathrm{m}^{2}$ to a patient with mesothelioma: the response rate is likely to be low $^{10}$ and toxicityespecially in patients with constitutional symptoms - can be appreciable. Choosing a control arm of limited efficacy may have made the pemetrexed and cisplatin combination appear more effective than it was. Indeed, from the quality of life data currently available, the symptom scores of patients treated with cisplatin $75 \mathrm{mg} / \mathrm{m}^{2}$ appeared to worsen on treatment, thus exaggerating the palliative benefit of the experimental treatment and emphasising why trials including a "no chemotherapy" arm may be appropriate.

Secondly, the investigators concluded that pemetrexed with cisplatin, folic acid and vitamin B12 should be the "standard front line therapy" for patients with malignant pleural mesothelioma. However, the data for patients given this exact combination showed that the improvement in overall survival compared with the questionable control arm only achieved borderline statistical significance $(p=0.051)$.
These criticisms weaken the argument that pemetrexed with cisplatin and vitamin supplementation should be standard treatment, although the combination is certainly an option for fitter patients. A randomised trial including a "no chemotherapy" arm remains reasonable, and the pilot phase of the UK trial has shown that patients are willing to be randomised into such a trial. The MSOl trial, with its comprehensive set of end points, should define the role of palliative chemotherapy in malignant pleural mesothelioma. It demands our full support, as do other trials examining new treatments for this once neglected group of cancer patients.

Thorax 2003;58:96-97

\section{Author's affiliation}

J P C Steele, Department of Medical Oncology, St Bartholomew's Hospital, London EC1A 7BE, UK; jeremy.steele@bartsandthelondon.nhs.uk

\section{REFERENCES}

1 Peto J, Decarli A, La Vecchia C, et al. The European mesothelioma epidemic. $\mathrm{Br} J$ Cancer 1999:79:666-72.

2 Peto J, Hodgson JT, Matthews FE, et al. Continuing increase in mesothelioma mortality in Britain. Lancet 1995;345:535-9.

3 Vogelzang NJ, J Rusthoven J, Paoletti $P$, et al Phase III single-blinded study of pemetrexed + cisplatin vs. cisplatin alone in chemonaive patients with malignant pleural mesothelioma. Proc Am Soc Clin Oncol 2002;21 :abstract 5. 4 Curtin NJ, Hughes AN. Pemetrexed disodium, a novel antifolate with multiple targets Lancet Oncol 2001:2:298-306.

5 Thodtmann R, Depenbrock $\mathrm{H}$, Blatter J, et al. Preliminary results of a phase I study with MTA (LY23 15 14) in combination with cisplatin in patients with solid tumors. Semin Oncol 1999;26(Suppl 6):89-93.

6 Manegold C, Gatzemeier U, von Pawel J, et al. Front-line treatment of advanced non-small-cell lung cancer with MTA (LY231514, pemetrexed disodium, ALIMTA) and cisplatin: a multicenter phase II trial. Ann Oncol 2000; 11:435-40.

7 Medical Research Council Clinical Trials Unit. www.ctu.ac.uk.

8 Steele JP, Shamash J, Evans MT, et al. Phase II study of vinorelbine in patients with malignant pleural mesothelioma. J Clin Oncol 2000;18:3912-7.

9 Middleton GW, Smith IE, O'Brien ME, et al. Good symptom relief with palliative MVP (mitomycin-C, vinblastine and cisplatin) chemotherapy in malignant mesothelioma. Ann Oncol 1998;9:269-73.

10 Mintzer DM, Kelsen D, Frimmer D, et al. Phase II trial of high-dose cisplatin in patients with malignant mesothelioma. Cancer Treat Rep 1985;69:711-2. 
Asthma guidelines

\section{The new BTS/SIGN asthma guidelines: where evidence leads the way}

B G Higgins, J G Douglas

\section{Announcing the publication of the new BTS/SIGN asthma guidelines as a supplement to this issue of Thorax.}

t is 12 years since the first British guidelines on asthma management in adults were published as two papers in the $B M J .{ }^{12}$ The British Thoracic Society (BTS) guidelines were rewritten in $1993^{3}$ with additional advice on childhood asthma, and further updated in 1995. ${ }^{4}$ Elsewhere, the Scottish Intercollegiate Guidelines Network (SIGN) published their guideline on the hospital management of asthma in $1996^{5}$ based on the BTS work, and subsequently published on the primary care management of asthma in $1998^{6}$ and the management of acute asthma in 1999. ${ }^{7}$ While the BTS versions have been among the most widely implemented of all clinical guidelines, there has been an increasing need to update them using evidence-based methodology and covering all aspects of asthma care. With this issue of Thorax the new British guidelines on the management of asthma ${ }^{8}$ produced jointly by the BTS and SIGN are published in a separate supplement. The development process has involved individuals from all relevant professional groups involved in asthma care in the UK. Initial literature searches based on key questions produced over 15000 abstracts and all relevant published papers up to the end of September 2001 were considered. What changes has this evidence-based review brought?

For those familiar with the previous guideline, the striking features will be the change in style and the increased size of the new version. The design will be more familiar to those working in Scotland since it follows the basic pattern of all SIGN publications. It is important - and hopefully interestingfor readers to be able to link the recommendations in the guideline to the supporting evidence, and the format of the guidelines follows naturally from this. Explanatory paragraphs citing the available evidence are accompanied by a clear recommendation. Although these are graded, it is worth emphasising that this reflects the strength of the evidence and not necessarily the importance of the recommendation. Those daunted by the size of the document will find that the recommendations are clearly highlighted, and anyone with a mind to do so will easily be able to pick their way through these and leave the supporting text for another day.

The guideline has grown bigger because several topics are covered in greater detail than previously and some new areas are included. There is a section addressing treatment of asthma by nonpharmacological methods including complementary medicine, acupuncture, homeopathy, and immunotherapy. This is an area of immense interest to many patients and a common reason for consultation with the UK National Asthma Campaign helpline; those practising more orthodox medicine need to be able to offer some guidance when patients ask advice about alternative treatments. There are also expanded sections on diagnosis and on the management of asthma in pregnancy. Some readers will feel that these contain nothing new, but there is still great concern about misdiagnosis of asthma, particularly in children, and evidence that patients with asthma tend to be undertreated when pregnant, indicating a need for guidance in these areas. The large bodies of literature on selfmanagement of asthma and organisation of care have also been explored and have led to some strong recommendations to implement measures which may not be current standard practice everywhere. Advice on the performance of high quality audit of asthma care is also included for the first time.

What key messages does the guideline offer and what has changed from the previous version? For many the nucleus of the guideline will be the advice on pharmacological management, particularly the treatment steps, and the section on management of acute asthma. The steps have been retained and there are still five in adults and older children (although consideration of step 3 suggests that this may be difficult to retain in future). There are, however, changes in the sequencing of treatment with a new emphasis in adults-and most particularly in children-on trials of other treatment before a patient reaches higher doses of inhaled steroid. This stems firstly from the evidence which shows that, although an inhaled steroid is the first choice preventative treatment in asthma, high doses are infrequently required; secondly, from concerns regarding the potential dangers of high doses, especially in children; and finally from the strong evidence of benefit from the introduction of long acting $\beta$ agonists at step 3 . The ceiling dose of inhaled steroid at step 3 has therefore been brought down to $800 \mu \mathrm{g}$ beclomethasone or equivalent in adults (compared with $2000 \mu \mathrm{g}$ in the previous BTS guideline) and $400 \mu \mathrm{g}$ in children. It is recommended that other agents, specifically a long acting $\beta$ agonist in the first instance, should be tried before exceeding these doses. For the management of acute asthma the eye catching changes are the inclusion of advice on intravenous magnesium as a treatment option for severe non-responding or life threatening attacks and the potential use of continuous nebulisation of $\beta$ agonists. No less important is the advice on identification of those patients at risk of life threatening asthma attacks.

Although these sections of the guideline are rightly regarded as important, it is to be hoped that the other sections are not dismissed as being of lesser value. Most paediatricians will tell cautionary tales of children in whom other diagnoses have been missed while the patient's asthma medication was remorselessly increased. Adult physicians too will recognise this scenario, hence the encouragement to seek objective support for the diagnosis of asthma and to review this when the response to treatment is poor. Similarly, many patients with asthma still do not have an agreed Asthma Action Plan (formerly known as a self-management plan), yet there is good evidence that doing so is of clinical benefit in terms of overall morbidity and in avoiding the need for hospitalisation. Action plans are best introduced as part of a structured educational package and practices which do not offer these should be encouraged to do so, particularly targeting patients whose treatment is at steps 3-5 and those who have had a recent hospital admission or $\mathrm{A} \& \mathrm{E}$ attendance. The literature on inhaler devices has also been reviewed. Metered dose inhalers, with or without spacers, have been shown to be as effective as other devices but, as ever, the pragmatic advice is to match the device to the patient on the basis of individual technique and preference.

At face value the section on nonpharmacological management offers no compelling reason for change from conventional practice. There is disappointingly little evidence of efficacy even for 
measures which many have long felt should be beneficial, such as reduction of exposure to house dust mite or avoidance of pet allergen. However, those reviewing this literature will feel that what is necessary is more conclusive evidence, be it positive or negative. This also applies to the data on alternative medical therapies where high quality studies are few. Even where there is good evidence of efficacy, recommendations are not necessarily clear cut. Immunotherapy can improve asthma but there are insufficient data to assess its value relative to conventional pharmacological treatment.

These are issues which require further research and, indeed, this is one of the great secondary benefits of producing formal evidence-based guidelines. The extensive review of the literature which is part of the process has revealed many areas where more evidence is needed before clear guidance can be given. The gaps in our knowledge are sometimes surprising. For example, despite the number of pharmacological studies carried out in asthma, we still do not know the threshold at which inhaled steroids should be introduced and we have no evidence to help decide which treatment strategy to try first at step 4. Some will regard it as a failure that there are not more grade A recommendations, but it would be better to regard this as a challenge. We have the opportunity to take this as a starting point from which to analyse the major gaps in our knowledge and develop appropriate research to address these. This process has already started with an initiative led by the Asthma Taskforce, administered by the National Asthma Campaign.

A further consideration for the future is the concept of developing a "living guideline". This would involve a regular-probably annual-review of the literature and revision of the guideline where appropriate. It is extremely difficult to keep a guideline both up to date and yet also grounded on firmly established evidence, but the current system of major revision every few years may lean too far away from the former aim.

In the meantime we believe that this new joint BTS/SIGN guideline represents the best synthesis of available evidence and practical advice on the clinical management of asthma. Implementing the recommendations should lead to improved care for our patients but, in addition, we would be delighted if this guideline acted as a stimulus to improving the evidence base available in the future.

Thorax 2003;58:98-99

\section{Authors' affiliations}

B G Higgins, Freeman Hospital, Newcastle upon Tyne, UK
J G Douglas, Aberdeen Royal Infirmary, Aberdeen, UK

Correspondence to: $\operatorname{Dr} B$ G Higgins, Freeman Hospital, High Heaton, Newcastle upon Tyne NE7 7DN, UK; b.g.higgins@ncl.ac.uk

\section{REFERENCES}

1 British Thoracic Society, Royal College of Physicians of London, King's Fund Centre, et al. Guidelines for the management of asthma in adults: I - Chronic persistent asthma. BM 1990;301:651-3.

2 British Thoracic Society, Royal College of Physicians of London, King's Fund Centre, et al. Guidelines for the management of asthma in adults: II - Acute severe asthma. BM 1990;301:797-800.

3 British Thoracic Society, British Paediatric Association, Royal College of Physicians of London, et al. Guidelines on the management of asthma. Thorax 1993;48(Suppl 2):S1-24.

4 British Thoracic Society, National Asthma Campaign, Royal College of Physicians of London, et al. The British guidelines on asthma management: 1995 review and position statement. Thorax 1997;52(Suppl 1):S1-21.

5 Scottish Intercollegiate Guidelines Network (SIGN). Hospital inpatient management of acute asthma attacks. Edinburgh: SIGN Publication No 6, 1996.

6 Scottish Intercollegiate Guidelines Network (SIGN). Primary care management of asthma. Edinburgh: SIGN Publication No 33, 1998.

7 Scottish Intercollegiate Guidelines Network (SIGN). Emergency management of acute asthma. Edinburgh: SIGN Publication No 38, 1999.

8 British Thoracic Society, Scottish Intercollegiate Guidelines Network (SIGN). British guideline on the management of asthma. Thorax 2003;58(Suppl I):i 1-94. 